\title{
Нефинансовая отчетность: международный контекст, российская практика
}

\author{
Елена Александровна Сенаторова \\ Кандидат экономических наук, доцент школы финансов, \\ факультет экономических наук, \\ Национальный исследовательский университет «Высшая школа экономики» \\ Москва, Мясницкая ул., 20 \\ финансовый директор ООО «Либра 36» \\ Москва, ул. Орджоникидзе, 7 \\ E-mail: senatorova.mail@gmail.com
}

\begin{abstract}
Аннотация
Целью исследования является оценка существующей практики реализации системы учета и отчетности в области устойчивого развития компаний, разработка предложений по совершенствованию нормативной базы в сфере нефинансовой отчетности (в рамках работы автора над международным научно-исследовательским проектом, University of Western Ontario, Canada). Неотъемлемой частью современного российского и международного бизнеса становится корпоративная социальная ответственность. Соответственно возрастают требования к качеству нефинансовой отчетности и учету. Детальный анализ российского и международного опыта, оценка актуальных трендов, на наш взгляд, будет способствовать повышению эффективности системы нефинансового учета и отчетности.

В статье представлен обзор последних тенденций в исследованиях, посвященных проблематике нефинансового учета и отчетности. Новизна статьи заключается в оценке и систематизации современных подходов к изучению проблем развития системы учета и отчетности в сфере устойчивого развития, предложены рекомендации по формированию нормативной базы в сфере нефинансовой отчетности.

Анализ показал, что для России наибольшее внимание следует уделять совершенствованию законодательной базы об индикаторах, предоставляемых в публичной нефинансовой отчетности, проблеме измерений разных форм капиталов, в том числе интеллектуального капитала, внесению таких индикаторов в регулирующие документы для реализации соответствующего федерального закона. Данный федеральный закон должен обеспечить предоставление публичной нефинансовой отчетности государственными корпорациями, публичными акционерными обществами, а также компаниями с существенным годовым объемом выручки и величиной активов.

Согласно результатам исследования наиболее прогрессивной формой публичной нефинансовой отчетности является интегрированная отчетность, концептуальный подход которой основан на интегрированном, системном мышлении. Системный подход к раскрытию нефинансовой информации создаст дополнительные возможности для анализа и совершенствования различных экономических, социальных и экологических аспектов развития российских и международных компаний. Соответственно, перспективным является формирование детализированного отечественного законодательства в сфере интегрированной отчетности в контексте реализации концепции устойчивого развития.
\end{abstract}

Ключевые слова: нефинансовая отчетность, устойчивое развитие, интегрированная отчетность, интеллектуальный капитал, глобальная инициатива по отчетности (GRI)

JEL: G30, Q56, Z00 


\section{Введение}

Международные и отечественные тенденции последних лет обусловливают активное развитие системы нефинансовой отчетности. Государство перестает играть основную роль в этом процессе, одновременно повышаются требования, связанные с устойчивым развитием компаний, со стороны крупнейших фондовых бирж, возрастают запросы в отношении публичной нефинансовой отчетности со стороны акционеров и инвесторов. По состоянию на апрель 2018 г. в крупнейшей электронной базе данных нефинансовых отчетов Corporate Register было зарегистрировано 95492 отчета, представленных 15896 организациями. Количество отчетов постоянно растет, в последние годы наблюдается снижение темпов роста [Corporate Register, 2018].

Развитие системы нефинансовой отчетности в России в целом соответствует международным тенденциям. К 2010 г. количество компаний, регулярно публикующих отчетность в сфере устойчивого развития, превышало 100. В настоящее время около 170 российских компаний реализуют концепцию устойчивого развития и публикуют соответствующую отчетность, общее количество опубликованных отчетов превысило 820. По данным Национального Регистра корпоративных нефинансовых отчетов и Библиотеки корпоративных нефинансовых отчетов Российского союза промышленников и предпринимателей по состоянию на 17 апреля 2018 г. за период, начиная с 2000 г., выпущено 73 экологических отчета, 311 социальных отчетов, 277 отчетов в области устойчивого развития, 141 интегрированный отчет, 26 отраслевых отчетов [РСПП, 2018].

Ситуация последних лет характеризуется равномерным ростом публикуемых нефинансовых отчетов. Тем не менее большинство крупнейших российских компаний не формируют нефинансовую отчетность, особенно это характерно для строительной отрасли, отрасли информационных технологий, ритейла, финансовой и телекоммуникационной сферы [Нефинансовая отчетность, 2017]. Напротив, наиболее активны в плане реализации концепции устойчивого развития компании атомной, энергетической, нефтегазовой, металлургической и химической отраслей [РСПП, 2018]. 10 из 20 крупнейших частных российских компаний, входящих в Рейтинг Forbes 2016 г., публикуют нефинансовую отчетность (в том числе ПАО «ЛУКОЙЛ, ОАО «Сургутнефтегаз», ПАО «Татнефть», ПАО «Северсталь», ПАО АНК «Башнефть») [Forbes, 2016].

\section{Обзор исследований}

Проблематика нефинансовой отчетности привлекает пристальное внимание исследователей уже более четверти века. Учет и отчетность - категории крайне консервативные, тем не менее развитию системы нефинансовой отчетности способствуют глобальные политические, экономические, социальные и эколо- гические изменения [Lehman, Kuruppu, 2017]. Многие современные исследователи считают, что мы стоим на пороге революции в финансовом и нефинансовом учете и отчетности [Chambers, 1999; Lehman, Kuruppu, 2017; Deegan, 2017]. Предлагаются новые информационные парадигмы развития учета и отчетности [Lev, $\mathrm{Gu}, 2016]$. Целесообразным представляется выделить четыре основных подхода к исследованию проблем развития системы нефинансовой отчетности.

Один из подходов к исследованию природы нефинансового учета и отчетности подразумевает рассмотрение этих систем в рамках концепции менеджериализма, в которой основными критериями успешности бизнеса являются показатели эффективности, именно на основании этих показателей принимаются основные управленческие решения. При данном подходе отличительной особенностью является отсутствие критики существующей системы нефинансовой отчетности в целях ее трансформации, обоснование стратегии прагматичной интеграции нефинансового учета и отчетности с бизнесом (с точки зрения управления) [Burritt and Schaltegger, 2010]. Внешняя среда, социальные и экологические системы рассматриваются с точки зрения полезности для бизнеса. Этот подход не допускает предположения о том, что природа имеет ценность сама по себе. Нефинансовая отчетность с этой точки зрения является лишь дополнительной информацией в общей системе корпоративной отчетности, позволяющей увеличить конкурентоспособность бизнеса, повысить его эффективность [Baker and Schaltegger, 2015]. Происходит адаптация исследований проблематики нефинансового учета и отчетности к существующим экономическим, бизнес-парадигмам, обосновывается внедрение новых технологий отчетности [Gray, 2013]. Эти исследования прежде всего нацелены на поиск возможностей, с помощью которых социальная и экологическая информация может способствовать совершенствованию бизнес-операций. Кроме того, работы в этой области позволяют выделять способы для получения информации различными группами внешних стейкхолдеров, а также в целях повышения эффективности процесса принятия внутренних управленческих решений на основе данных нефинансовой отчетности. Исследователи отмечают, что при использовании данного подхода представление нефинансовой информации в соответствующей отчетности безусловно будет меняться, но фокус в ближайшее время останется на процедурах представления нефинансовой отчетности как отражении влияния неолиберальных идеологических принципов и точек зрения [Lehman, Kuruppu, 2017].

Эволюционные теории развития нефинансовой отчетности подразумевают совершенствование экологических систем, развитие социальных отношений в соответствии с существующими социально-экономическими системами [Contrafatto, Thomson, and Monk, 2015; Thomson, 2015]. В основе данного подхода лежит 
утверждение об отличительной нравственно-этической природе исследований в области нефинансового учета и отчетности. Социальные изменения возникают на базе существующей системы посредством этических и моральных трансформаций, на которые влияют различные культуры. Это улучшает этические результаты взаимодействия людей друг с другом, положительно влияет на социальные и экологические системы [Lehman, Kuruppu, 2017]. Многие современные ученые также исследуют роль бизнеса в развитии общественных отношений [Walker, 2016], природу взаимоотношений между бизнесом и государственными институтами, обладающими потенциалом информировать людей, для которых окружающая среда (природа) является важнейшим ресурсом [Arrington, Francis, 1989, 1993; Arrington, Schweiker, 1992; Lehman, 2013, 2017]. Эти работы нацелены на понимание природы социального учета, на исследование того, как различные сценарии нефинансового учета и учетных практик могут быть эффективно интегрированы в трансформационные программы устойчивого развития, как нефинансовая информация может влиять на социальные изменения. Авторы также фокусируются на микрополитических изменениях, которые влияют на изменение отношений в обществе [Gray, Brennan, Malpas's, 2014]. Исследуются причины того, что концепция устойчивого развития часто «пробуксовывает» в добывающих компаниях, не совпадая с целями и возможностями данных компаний. Наиболее актуальным моментом эволюционных теорий продолжает оставаться вопрос о том, что же означает социальная ответственность компании [Harvey, 2014].

Существуют также радикальные теории, основанные на критике современной системы нефинансовой отчетности и социально-экономических отношений, лежащих в основе ее формирования. При этом не стоит забывать, что те идеи, которые ранее казались радикальными (например, идеи марксизма), теперь уже классика, воплощенная на практике. Осуществляется критика существующих моделей, идеи нерегулируемой максимизации прибыли в среднесрочной и долгосрочной перспективах, неравенства в современном обществе, а также тех основ, на которых формируется нефинансовая отчетность: политических процессов, базирующихся на политической и социальной природе капиталистических отношений с учетом таких глобальных социофилософских течений, как марксизм и постмарксизм [Tinker, Neimark, Lehman, 1991]. Исследователи также подчеркивают, что прежде всего необходимо совершенствовать нормативную базу с целью предотвращения деятельности компаний, противоречащей основам устойчивого развития. Роль нормативных документов при этом рассматривается в контексте совершенствования социальноэкономических отношений. Вместо концентрации на экоэффективности, измерении показателей, процедурах отчетности, управлении финансовым, социальным, экологическим влиянием компаний ряд ученых предлагает пересмотреть значение и смысл понятия «успех» и на этой основе исследовать природу контроля за устойчивым развитием компаний [Milne, Gray, 2013]. При этом подчеркивается, что нормативная база должна выстраиваться на основе прогрессивных учетных практик и исследований [Spence et al., 2013; Gray et al., 2014; Buhr, Gray, Milne, 2014]. Часть исследователей предлагает искать пути решения проблем в рамках существующей социальной системы. Действительно, существующая система, которая привела к экологическому кризису, с очень небольшой долей вероятности сможет найти пути его преодоления; менять или кардинально реформировать необходимо саму систему [Lehman, 2015].

Четвертый подход к исследованию проблем развития системы нефинансовой отчетности подразумевает интерпретацию, изучение природы нефинансового учета, выявление особенностей взаимодействия социальной, экологической и экономической систем, что позволило выдвинуть гипотезу, согласно которой нефинансовая отчетность пошагово позволяет компаниям придерживаться принципов устойчивого развития. Необходимо не только интегрировать устойчивое развитие в бизнес, но прежде всего сделать так, чтобы каждый менеджер думал о том, как реализовывать в своей работе концепцию устойчивого развития [Higgins, Coffey, 2016]. Ряд исследователей иллюстрируют ограничения реализации системы нефинансовой отчетности и соответственно принимаемых на ее основе решений [Brown, Dillard, 2015]. При этом акцентируется, что необходимо внедрять такую систему нефинансовой отчетности, которая будет в наибольшей степени соответствовать природе существующих экологических систем [Lehman, 2015]. Наиболее прогрессивные, на наш взгляд, исследования концентрируются на изучении природы «неустойчивого» развития, перспектив развития нефинансового учета и отчетности, роли учета в изменении мировой экономики [Gray, 2016; Milne, Gray, 2013].

Таким образом, по мнению многих ученых, за последние несколько лет был достигнут значительный прогресс в исследовании развития системы нефинансового учета и отчетности, тем не менее существует огромный потенциал относительно будущих исследований по данной проблематике [Parker, 2011; Owen, 2008; Deegan, 2017; Lehman, Kuruppu, 2017].

\section{Создание глобальных инициатив}

Основной тенденцией последних лет в международной практике является создание эффективных инициатив и организаций в сфере устойчивого развития и нефинансовой отчетности. В 2016 г. произошло знаковое событие - вступила в силу Повестка дня до 2030 года в области устойчивого развития Организации Объединенных Наций. Она содержит 17 целей (SDGS - Sustainable development goals), имеющих количественное выражение и направленных прежде 
всего на сохранение ресурсов Земли, обеспечение всеобщего благополучия и гармоничного развития. В рамках концепции устойчивого развития предполагается разрешить противоречия, сопровождающие взаимоотношения природы и общества, настоящего и будущих поколений [UNDP, 2018].

В 2016 г. произошло еще одно важное событие, непосредственно связанное с реализацией глобальных целей устойчивого развития: было подписано Парижское соглашение по изменению климата, приняты обязательства по разработке мер, предотвращающих повышение общемировой температуры более чем на два градуса Цельсия. Парижское соглашение представляет собой «дорожную карту» мер, позволяющих сократить выбросы и снизить риски, связанные с изменением климата. Ратификация Парижского соглашения Россией планируется не ранее 2019 г. В случае его нератификации, по мнению ряда экспертов, риски для российских экспортеров могут возрасти [Ответственная деловая практика, 2017].

В последние годы были предприняты другие важные международные инициативы. Так, в 2009 г. была создана United Nations Sustainable Stock Exchanges Initiative (SSE Initiative), регулярно раскрывающая информацию об особенностях существующих практик в области устойчивого развития. По итогам 2017 г. 85 бирж реализуют цели устойчивого развития, многие из них выпустили соответствующие нормативные документы по нефинансовой отчетности. К сожалению, Московская биржа не входит в этот список. Наиболее эффективно биржи реализуют такие цели устойчивого развития, как борьба с изменениями климата, ответственное потребление и производство, гендерное равенство, партнерство в интересах устойчивого развития [SSE Initiative, 2018]. Информация к раскрытию, запрашиваемая биржами - участницами данной организации от компаний, включает прежде всего цели компании в сфере устойчивого развития, потребление водных ресурсов, выбросы в атмосферу загрязняющих веществ.

Функционирование G20 Green Finance Study Group также способствует реализации концепции устойчивого развития, расширяет возможности финансирования соответствующих проектов. В 2017 г. деятельность группы была сконцентрирована на двух основных направлениях: ERA (environmental risk analysis) в финансовой отрасли и $P A E D$ (publicly available environmental data) - использование соответствующих данных в финансовом анализе [G20, 2017]. Основной целью группы является определение институциональных и рыночных барьеров зеленому финансированию и изучение возможностей финансовой системы мобилизовать частный капитал для зеленых инвестиций, опираясь на опыт различных стран.

Деятельность созданной в 2007 г. инициативы PRI (Principles for Responsible Investment) сосредоточена на развитии финансовой системы, соответствующей принципам устойчивого развития (SFS - sustainable financial system). По итогам 2017 г. количество компаний - участниц данной инициативы превысило 1700. $P R I$ эффективно реализует проекты, соответствующие целям ответственного инвестирования, объединяющие интересы различных групп инвесторов, рейтинговых агентств.

Необходимость изменений в законодательстве ряда стран Европейского союза была продиктована принятой Directive 2014/95/EU (далее - Директивой). Компаниям со среднесписочной численностью сотрудников 500 и более человек в обязательном порядке необходимо раскрывать нефинансовую информацию, связанную с экологическими и социальными аспектами, предписанными этой Директивой [DIRECTIVE, 2014]. Компании также обязаны раскрывать нефинансовые ключевые показатели эффективности (KPI Key Performance Indicators). Новые требования к раскрытию данных направлены прежде всего на унификацию и повышение прозрачности нефинансовой отчетности.

Кроме того, важнейшими инициативами стали принятие Повестки по финансированию для развития (Action Agenda for Financing for Development), создание Рабочей группы по зеленому финансированию (Green Finance Study Group) и Плана действий по раскрытию финансовой информации по изменению климата (Task Force on Climate-related Financial Disclosures by the Financial Stability Board), появление рабочей группы по раскрытию финансовой информации, связанной с изменением климата (Financial Stability Board's Task Force on Climate-Related Financial Disclosures) [Ответственная деловая практика, 2017]. Обобщая вышесказанное, важно отметить, что рост количества инициатив, разработка нормативной базы в данной сфере в последние годы говорит о возрастающем внимании со стороны общества, бизнеса, государственных институтов к проблемам социальной и экологической безопасности, устойчивого развития, совершенствования нефинансовой отчетности.

\section{Развитие стандартов нефинансовой отчетности}

В сфере нефинансовой отчетности не существует единого подхода к предоставлению информации, тем не менее создаются международные и отечественные стандарты и рекомендации, в том числе с целью обеспечения сопоставимости отчетов различных компаний. Наиболее часто используемыми являются следующие нормативные документы: стандарты GRI, $S A S B$, стандарты серий ISO, AA 1000, Рамочное руководство по интегрированной отчетности, Глобальный договор ООН, Рамочное руководство по экологической отчетности, Протокол по естественному капиталу, Социальная хартия российского бизнеса.

На наш взгляд, наиболее детальные рекомендации в отношении элементов нефинансовой отчетности и используемых показателей содержатся в стандартах 
GRI [GRI, 2018]. Большинство российских компаний применяют именно этот стандарт. В разработанной несколько лет назад новой версии GRI G4 подчеркивается, что стандарт стал удобнее в использовании, чем предыдущие его редакции, более того, в нем делается акцент на темах, наиболее существенных для бизнеса и общества. Данная версия является наиболее популярной в настоящее время. В 2016 г. GRI представила модульные стандарты. Была изменена структура стандартов нефинансовой отчетности: предлагаются три универсальных стандарта (GRI 101: Основные стандарты, GRI 102: Общие показатели отчетности, GRI 103: Подход в области менеджмента) и 33 специфических стандарта, сгруппированных в три серии (Серия 200 «Экономические стандарты», Серия 300 «Экологические стандарты», Серия 400 «Социальные стандарты»). Компании имеют право выбирать те специфические стандарты, которые способствуют раскрытию наиболее существенных аспектов. Компании также вправе публиковать либо полноценный отчет, либо выборочные данные по определенным специфическим стандартам с указанием того, какие именно стандарты и показатели использовались. Модульные стандарты упрощают процесс публикации отчета компаниями - «новичками» в этой сфере, предоставляя возможность поэтапного раскрытия нефинансовой информации.

Популярными прежде всего среди публичных компаний США и компаний, акции которых котируются на американских фондовых биржах, являются стандарты, разработанные Sustainable Accounting Standards Board (SASB). Детально разработанные стандарты позволяют отражать нефинансовую информацию в формах 10-K, 20-F, 40-F в соответствии с законодательством США. С каждым годом растет количество сертифицированных бухгалтеров в сфере устойчивого развития (FSA - Fundamentals of Sustainability Accounting Credential) [SASB, 2018].

Последние изменения стандартов серии $A A 1000$ направлены прежде всего на установление более тесной взаимосвязи ключевых принципов концепции устойчивого развития, применении прогрессивных международных практик в данной сфере.

В 2010 г. был опубликован ISO 26000: международный стандарт, содержащий руководство о том, как компании могут работать социально ответственным образом [ISO, 2018]. ISO 26000 содержит определение социальной ответственности и других важнейших категорий, принципы социальной ответственности и подробные рекомендации по их реализации. В последние годы также активно выпускаются ISO 14001: международные стандарты по управлению окружающей средой, содержащие требования соблюдения компаниями ограничений на использование энергии, выбросов углерода, уменьшение отходов, сокращение использования воды. Данные стандарты также являются признанными лидерами в области экологии на международном уровне [ISO, 2018].
Трендом последних лет стал рост количества и качества стандартов и руководств в сфере нефинансовой отчетности. При этом GRI продолжает оставаться лидером среди используемых платформ нефинансовой отчетности, сочетая не только учетные, но и аналитические функции. GRI реализует стратегию, предусматривающую установление партнерских отношений с другими платформами отчетности в области устойчивого развития и использование компаниями различных систем показателей.

\section{Интегрированное мышление и интегрированная отчетность}

Важной тенденцией сегодня является развитие системы интегрированной отчетности, перспективной с точки зрения интегрированного мышления, глобализации, комплексного подхода к данным, систематизации информации компаний, инвестиционной привлекательности. Интегрированная отчетность призвана объединить финансовую и нефинансовую отчетность, публиковать информацию в более удобном для инвесторов формате. Важнейшей задачей интегрированной отчетности является развитие интегрированного мышления.

В международной практике интегрированные отчеты были впервые опубликованы в середине 2000-х гг. В 2010 г. был образован Международный Совет по интегрированной отчетности. В этом же году компаниям, акции которых котируются на фондовой бирже Йоханнесбурга (ЮАР), было предписано предоставлять интегрированную отчетность. Количество интегрированных отчетов крупнейших компаний продолжает расти. Среди партнеров Международного Совета по интегрированной отчетности: Association of Chartered Certified Accountants (ACCA), Chartered Institute of Management Accountants (CIMA), International Federation of Accountants (IFAC), IFRS Foundation, Sustainability Accounting Standards Board, Global Reporting Initiative, World Business Council for Sustainable Development [IIRC, 2018]. В отечественной практике соответствующие исследования проводятся, и развитие системы отчетности происходит с участием Российской региональной сети (РPC) по интегрированной отчетности, созданной в 2012 г. по инициативе Государственной корпорации по атомной энергии «Росатом» при поддержке Международного совета по интегрированной отчетности [РРС, 2018].

Концепцию интегрированной отчетности поддерживают крупнейшие компании, входящие в пилотную программу при Международном Совете по интегрированной отчетности: Danone, Coca-Cola, Deloitte, KPMG, HSBC, Unilever, Microsoft, Volvo. Отечественные компании (Государственная корпорация по атомной энергии «Росатом», ПАО «НК «Роснефть», К «УРАЛСИБ») также участвуют в пилотном проекте [IR, 2018]. Вызовы и риски, связанные с формированием системы интегрированной отчетности, 
включают прежде всего недостаток доверия к отчетам со стороны инвесторов, неразработанность некоторых аспектов, сложность в их понимании, что ведет к медленным темпам развития.

\section{Отечественные тенденции в сфере нефинансовой отчетности}

В отечественной практике наиболее актуальным документом в плане реализации концепции устойчивого развития стало подписанное Председателем Правительства Российской Федерации 5 мая 2017 г. Распоряжение об утверждении Концепции развития публичной нефинансовой отчетности и плана мероприятий по реализации Концепции развития публичной нефинансовой отчетности (далее - Концепция). Появлению данной концепции предшествовало принятие в 2012 г. Директивы Председателя Правительства РФ В.В. Путина, предписывающей государственным компаниям (22 компании с государственным участием) необходимость предоставления нефинансовой отчетности. В Концепции отмечается, что «стимулирующую роль в развитии публичной нефинансовой отчетности может оказать резолюция Организации Объединенных Наций, содержащая 17 целей в сфере устойчивого развития и определяющая, с одной стороны, необходимость разработки системы показателей с целью мониторинга достижения этих целей как на национальном, так и на глобальном уровне, с другой стороны, включающая задачи по отражению вклада организаций в устойчивое развитие, решаемые в рамках публичной нефинансовой отчетности» [Концепция, 2017]. Публичная нефинансовая отчетность раскрывает информацию о деятельности в области устойчивого развития, отражает взаимосвязь экономических, социальных и экологических аспектов. Концепция направлена прежде всего на развитие системы стимулирования компаний к росту прозрачности и информационной открытости результатов влияния их деятельности на окружающую среду и общество, в разрезе экономической, социальной и экологической составляющих; повышение информированности о международных стандартах и инициативах в области устойчивого развития. Среди основных задач Концепции важно отметить создание фундамента для формирования нормативно-правовой базы по вопросам устойчивого развития; поддержка распространения и использования в деятельности компаний единых понятий в сфере соответствующей отчетности; содействие повышению квалификации и развитию профессиональной подготовки в сфере устойчивого развития. Наиболее значимым моментом в этой связи является конкретный План мероприятий по реализации указанных задач. Реализация Концепции предусматривает несколько этапов. На первом этапе (2017-2018) приоритетным является развитие нормативной и методической базы; определение состава базовых показателей, необходимых для раскрытия информации о деятельности компаний в экономической, социальной и экологической сферах; определение перечня компаний, для которых требование публикации нефинансовой отчетности будет обязательным. На втором этапе (2019-2020) планируется дальнейшее развитие нормативной и методической базы, внедрение стимулирующих механизмов; развитие системы повышения квалификации специалистов, совершенствование образовательных программ; распространение требований обнародования результатов деятельности в области устойчивого развития прежде всего на государственные компании. На третьем этапе (2021-2022) предусматривается внедрение требования в отношении параметров подтверждения, проверки соответствующей отчетности; в состав компаний, на которые распространяется требование публикации отчетности, предполагается включить акционерные общества, ценные бумаги которых котируются на биржах. На четвертом этапе (с 2023 г.) планируется распространить требование публикации отчетности в области устойчивого развития и прочих видов публичных нефинансовых отчетов на 500 крупнейших российских компаний, объем выручки которых соответствует критериям ведущих российских рейтингов [Концепция, 2017].

Другим важнейшим документом, принятым в последние годы, стал Кодекс корпоративного управления, одобренный советом директоров Банка России 21 марта 2014 г. и содержащий рекомендации прежде всего крупным публичным частным и государственным компаниям. В Кодексе корпоративного управления в том числе содержатся рекомендации по раскрытию информации в сфере экологической и социальной ответственности. Такая информация может включать описание политики компании в социальной и экологической сфере, а также отчетность о деятельности в области устойчивого развития, составленную в соответствии с международными стандартами нефинансовой отчетности.

В отечественной практике экологическая отчетность является обязательной для предоставления в государственные органы статистики, но не публичной. В 2012 г. были приняты «Основы государственной политики в области экологического развития Российской Федерации на период до 2030 года» (утверждены Президентом РФ 30.04.2012), предусматривающие развитие нефинансовой отчетности на добровольной основе, постепенный переход к обязательной публикации соответствующей заверенной отчетности компаниями с государственным участием. В дальнейшем были приняты важнейшие нормативные акты, реализация которых должна изменить ситуацию в экологической сфере: постановление Правительства РФ от 15.04.2014 № 326 «Об утверждении государственной программы Российской Федерации “Охрана окружающей среды” на 2012-2020 годы» и Указ Президента РФ от 19.04.2017 № 176 «О Стратегии экологической безопасности Российской Федерации на период до 2025 года». 


\section{Выводы}

Несмотря на трансформацию общественного мнения и значительные перемены в области устойчивого развития, изменения в сфере нефинансовой отчетности происходят очень медленно. Существует множество точек зрения о том, какие изменения будут происходить в этой области в ближайшие годы: будут меняться подходы к сбору и анализу данных в сфере устойчивого развития; нефинансовая отчетность будет представлять собой информацию в режиме реального времени, поступающую из различных источников. Проблематика, определяющая будущее нефинансовой отчетности, включает прежде всего 17 целей устойчивого развития, глобальные вызовы: рост населения и неравенства доходов, защита прав человека, изменение климата, охрана экосистем, управление эффективностью бизнеса, цифровизация экономики [Lehman, Kuruppu, 2017; Deegan, 2017; Ответственная деловая практика, 2017]. Развитие единой системы международных стандартов нефинансовой отчетности, отражающих отраслевую специфику, позволит использовать нефинансовую информацию в управлении бизнес-процессами, будет способствовать формированию бизнес-моделей, нацеленных на устойчивое развитие.

В международной практике наблюдается рост количества заверенных нефинансовых отчетов. В России по итогам 2016 г. половина компаний, выпускающих нефинансовую отчетность, не использовала форму внешнего заверения отчетов. Сохраняется тренд наибольшей востребованности общественного заверения Советом нефинансовой отчетности РСПП (примерно $20 \%$ от общего числа отчетов). Происходит увеличение количества компаний, использующих несколько типов проверки: например, со стороны профессиональных аудиторов и РСПП [РСПП, 2018]. В целом отечественные тенденции в сфере развития системы независимого заверения отчетности соответствуют международным трендам.

Как нам кажется, эффективное формирование системы нефинансовой отчетности невозможно без повышения уровня образованности в данной сфере. Целесообразным представляется введение обязательного курса «Нефинансовая отчетность» на программах магистратуры при получении экономического образования, развитие системы сертификации соответствующих специалистов.

В соответствии с проведенным анализом важно подчеркнуть, что необходимо дальнейшее совершенствование нормативной базы в отношении оцифровки капиталов, в том числе интеллектуального капитала [Feruleva, Ivashkovskaya, 2018], в контексте устойчивого развития компаний, данных об основных показателях, предоставляемых в отчетности в сфере устойчивого развития. Кроме того, в этой связи важным является скорейшее принятие федерального закона, обеспечивающего раскрытие соответствующей отчет- ности государственными корпорациями, публичными акционерными обществами, а также компаниями с годовым объемом выручки или величиной активов в размере 5 млрд руб. и более.

Одним из трендов в международной практике становится рост количества компаний, раскрывающих в нефинансовой отчетности данные по ключевым показателям эффективности (KPI) в сфере устойчивого развития, таких как рост энергоэффективности; снижение потребления воды; сокращение выбросов в результате модернизации оборудования и внедрения производственных технологий, соответствующих принципам устойчивого развития. Важным моментом является дальнейшее расширение и совершенствование отечественных методик по раскрытию информации о ключевых показателях эффективности в нефинансовой отчетности, соответствующих прогрессивным международным практикам [Ivashkovskaya, Stepanova, Eliseeva, 2014].

Наиболее заметной тенденцией последних лет в отечественной и международной практике стало повышение интереса к интегрированной отчетности, комплексному подходу к раскрытию информации о деятельности компании. При переходе на интегрированный формат отчетности важным является минимизация рисков, связанных с сокращением существенной информации, влияющей на качество публикуемых отчетов. В этой связи целесообразным является развитие системы нормативного регулирования в сфере интегрированной отчетности в международной и отечественной практике.

Как показывает проведенное исследование, развитие системы нефинансовой отчетности предполагает совершенствование международного и российского законодательства в соответствии с актуальными тенденциями, внедрение прогрессивных практик с учетом системного подхода.

\section{Список литературы}

1. Борзаков Д.В. Институционализация корпоративной нефинансовой отчетности в России и за рубежом. Вестник Воронежского государственного университета. Серия: Экономика и управление. 2015;(3):58-66.

2. Концепция развития публичной нефинансовой отчетности и плана мероприятий по реализации Концепции развития публичной нефинансовой отчетности. Утверждены распоряжением Правительства Российской Федерации от 5.05.2017 года № 876-p. URL: http://docs.cntd.ru/ document/456064017 (дата обращения: 17.03.2018).

3. Макаров И. Климат будущего: почему России стоит ратифицировать Парижское соглашение. 2016. URL: http://www.rbc.ru/opinions/economics/04/11/2016/581b6 a5c9a7947574f524263 
4. Нефинансовая отчетность компаний: в погоне за успехом. Информационный бюллетень. Чистые технологии и устойчивое развитие. 2017;(2). URL: http://www.ey.com/Publication/vwLUAssets/EYccass-newsletter-may-2017/\$File/EY-ccass-newslettermay-2017.pdf (дата обращения: 16.02.2018)

5. Ответственная деловая практика в зеркале отчетности: настоящее и будущее. Аналитический обзор корпоративных нефинансовых отчетов: 20152016 годы выпуска. М.: РСПП; 2017. 140 c. URL: http:// media.rspp.ru/document/1/7/4/743222fc4c6650093518c6 35d0e8ecdd.pdf (дата обращения: 7.03.2018).

6. РИА Рейтинг. 2017. URL: http://www.riarating.ru/ infografika/20160127/630007042.html

7. Российская Региональная Сеть (РРС) по ИО. 2018. URL: http://ir.org.ru/regionalnaya-set

8. Российский союз промышленников и предпринимателей (РСПП). 2018. URL: http://rspp.ru/

9. Шесть тенденций в области устойчивого развития компаний в 2013 году. Исследование, проведенное компанией EY совместно с GreenBiz Group. URL: http://green.ucci.org.ua/wp-content/uploads/2016/11/EYSix-growing-trends-in-sustainability-2013-RUS.pdf (дата обращения: 16.02.2018).

10. Урсул А.Д. Концептуальные проблемы устойчивого развития. Использование и охрана природных ресурсов в России. 2005;(1):30-38.

11. Arrington C.E., Francis J.R. Letting the chat out of the bag: Deconstruction, privilege and accounting research. Accounting, Organizations and Society. 1989;14(1-2):128. DOI: 10.1016/0361-3682(89)90030-5

12. Arrington C.E., Francis J.R. Giving economic accounts: Accounting as cultural practice. Accounting, Organizations and Society. 1993;18(2-3):107-124. DOI: 10.1016/0361-3682(93)90029-6

13. Arrington C.E., Schweiker W. The rhetoric and rationality of accounting research. Accounting, Organizations and Society. 1992;17(6):511-533. DOI: 10.1016/0361-3682(92)90011-G

14. Baker M., Schaltegger S. Pragmatism and new directions in social and environmental accountability research. Accounting, Auditing \& Accountability Journal. 2015;28(2):263-294. DOI: 10.1108/AAAJ-08-2012-01079

15. Brown J., Dillard J. Opening accounting to critical scrutiny: Towards dialogic accounting for policy analysis and democracy. Journal of Comparative Policy Analysis: Research and Practice. 2015;17(3):247-268. DOI: 10.1080/13876988.2014.989684

16. Buhr N., Gray R., Milne M.J. Histories, rationales, voluntary standards and future prospects for sustainability reporting: CSR, GRI, IIRC and beyond. In: Bebbington J., Unerman J., O'Dwyer B., eds. Sustainability accounting and accountability. Abingdon, New York: Routledge; 2014:51-71.
17. Burritt R.L., Schaltegger S. Sustainability accounting and reporting: Fad or trend? Accounting, Auditing \& Accountability Journal. 2010;23(7):829-846. DOI: 10.1108/09513571011080144

18. Chambers R.J. The poverty of accounting discourse. Abacus. 1999;35(3):241-252. DOI: 10.1111/14676281.00044

19. Contrafatto M., Thomson I., Monk E.A. Peru, mountains and los niños: Dialogic action, accounting and sustainable transformation. Critical Perspectives on Accounting. 2015;33:117-136. DOI: 10.1016/j. cpa.2015.04.009

20. Corporate Register. 2018. URL: http://www. corporateregister.com

21. Deegan C. Twenty five years of social and environmental accounting research within Critical Perspectives of Accounting: Hits, misses and ways forward. Critical Perspectives on Accounting. 2017;43:6587. DOI: $10.1016 /$ j.cpa.2016.06.005

22. Directive 2014/95/EU of the European Parliament and of the Council of 22 October 2014. URL: http://eur-lex.europa.eu/legal-content/EN/ TXT/?uri=CELEX\%3A32014L0095

23. Feruleva N.V., Ivashkovskaya I.V. Assessment of intellectual capital influence on corporate value as a field for further investigations in corporate finance. Korporativnye finansy $=$ Journal of Corporate Finance Research. 2018;15(1):64-76.

24. Forbes. 2016. URL: http://www.forbes.ru

25. Global Reporting Initiative (GRI). 2018. URL: https:// www.globalreporting.org

26. Gray R., Brennan A., Malpas J. New accounts: Towards a reframing of social accounting. Accounting Forum. 2014;38(4):258-273.

DOI: $10.1016 /$ j.accfor.2013.10.005

27. Gray R. Back to basics: What do we mean by environmental (and social) accounting and what is it for? - A reaction to Thornton. Critical Perspectives on Accounting. 2013;24(6):459-468. DOI: 10.1016/j. cpa.2013.04.005

28. Gray R. Reading for displeasure: Why bother with social accounting at all? Social and Environmental Accountability Journal. 2 016;36(2):153-161.

DOI: 10.1080/0969160X.2016.1197625

29. G20 green finance synthesis report 2017.

URL: http://unepinquiry.org/wp-content/ uploads/2017/07/2017_GFSG_Synthesis_Report_EN.pdf

30. Harvey B. Social development will not deliver social licence to operate for the extractive sector. The Extractive Industries and Society. 2014;1(1):7-11. DOI: 10.1016/j.exis.2013.11.001 
31. Higgins C., Coffey B. Improving how sustainability reports drive change: A critical discourse analysis. Journal of Cleaner Production. 2016;136(Pt. A):18-29. DOI: 10.1016/j.jclepro.2016.01.101.

32. International Integrated Reporting Council (IIRC). 2018. URL: http://integratedreporting.org

33. International Organization for Standardization (ISO). 2018. URL: https://www.iso.org/

34. Ivashkovskaya I.V., Stepanova A.N., Eliseeva N. Does corporate financial architecture contribute to sustainable corporate growth? The evidence from Russian companies. Korporativnye finansy = Journal of Corporate Finance Research. 2014;8(4):11-33.

35. Kuzubov S.A. Sustainability: A new paradigm of corporate reporting. Korporativnye finansy $=$ Journal of Corporate Finance Research. 2017;14(4):7-10.

36. Lehman G., Kuruppu S.C. A framework for social and environmental accounting research. Accounting Forum. 2017;41(3):139-146. DOI: 10.1016/j.accfor.2017.07.001

37. Lehman G. Moral will, accounting and the phronemos. Critical Perspectives on Accounting. 2014;25(3):210-216. DOI: 10.1016/j.cpa.2013.10.004

38. Lehman G. Charles Taylor's ecological conversations: politics, commonalities and the natural environment. London: Palgrave Macmillan; 2015. 201 p.

39. Lehman G. The language of environmental and social accounting research: The expression of beauty and truth. Critical Perspectives on Accounting. 2017;44:30-41. DOI: 10.1016/j.cpa.2016.11.005

40. Lev B., Gu F. The end of accounting and the path forward for investors and managers. Hoboken, NJ: John Wiley \& Sons, Inc.; 2016. 288 p.

41. Milne M.J., Gray R.H. W(h)ither ecology? The triple bottom line, the global reporting initiative, and corporate sustainability reporting. Journal of Business Ethics. 2013;118(1):13-29. DOI: 10.1007/s10551-012-1543-8

42. Owen D. Chronicles of wasted time?: A personal reflection on the current state of, and future prospects for, social and environmental accounting research. Accounting, Auditing \& Accountability Journal. 2008;21(2):240-267. DOI: 10.1108/09513570810854428
43. Parker L.D. Building bridges to the future: Mapping the territory for developing social and environmental accountability. Social and Environmental Accountability Journal. 2011;31(1):7-24. DOI: 10.1080/0969160X.2011.556389

44. Spence C. Social accounting's emancipatory potential: A Gramscian critique. Critical Perspectives on Accounting. 2009;20(2):205-227. DOI: 10.1016/j. cpa.2007.06.003

45. Spence C., Chabrak N., Pucci R. Doxic sunglasses: A response to "Green accounting and Green Eyeshades: Twenty years later". Critical Perspectives on Accounting. 2013;24(6):469-473. DOI: 10.1016/j.cpa.2013.05.002

46. Sustainability Accounting Standards Board (SASB). 2018. URL: https://www.sasb.org/

47. Sustainable Stock Exchanges Initiative (SSE Initiative). 2018. URL: http://www.sseinitiative.org

48. The road ahead. The KPMG survey of corporate responsibility reporting 2017. URL: https://assets.kpmg. com/content/dam/kpmg/xx/pdf/2017/10/kpmg-surveyof-corporate-responsibility-reporting-2017.pdf

49. Thomson I. 'But does sustainability need capitalism or an integrated report' a commentary on 'The International Integrated Reporting Council: A story of failure' by Flower, J. Critical Perspectives on Accounting. 2015;27:1822. DOI: $10.1016 /$ j.cpa.2014.07.003

50. Tinker T., Neimark M., Lehman C. Falling down the hole in the middle of the road: Political quietism in corporate social reporting. Accounting, Auditing \& Accountability Journal. 1991;4(2):28-54. DOI: 10.1108/09513579110000504

51. United Nations Development Programme (UNDP). 2018. URL: http://www.undp.org

52. Walker S.P. Revisiting the roles of accounting in society. Accounting, Organizations and Society. 2016;49:41-50. DOI: 10.1016/j.aos.2015.11.007 


\title{
Non-financial Reporting: International Context, Russian Practice
}

\author{
Elena Senatorova \\ $\mathrm{PhD}$, Associate Professor of School of Finance \\ Department of Finances, National Research University Higher School of Economics, \\ 20, Myasnitskaya st., Moscow, Russia \\ CFO, Libra 36 Ltd \\ 7 Ordzhonikidze, Moscow, Russia \\ E-mail: senatorova.mail@gmail.com
}

\begin{abstract}
The main aim of the research is to analyze the existing sustainability accounting and reporting practices, to set up recommendations for improving the sustainability reporting legislation (as a result of author's participation in the international research project, University of Western Ontario, Canada). Corporate social responsibility has become part and parcel of Russian business. Consequently, the demand to the quality of non-financial accounting and reporting has increased. Detailed analysis of Russian and international experience, assessment of current trends contributes to an improvement of the non-financial accounting and reporting system.

The article includes literature review of research in non-financial reporting problematic. Particular attention is paid to current research trends. The paper contains of the acquired analysis and classification of recent themes in non-financial reporting and accounting research.

Based on this analysis, we conclude that the general attention should be paid to the improvement of legislation in the field of non-financial reporting regarding the information related to the indexes disclosed in the non-financial reports, capital data, including intellectual capital in term of companies' sustainable development. The compulsory regulation of non-financial reporting and accounting is required for state companies, joint-stock companies, companies with large sales and assets.

According to research, the most advanced form of non-financial reporting is integrated reporting. Conceptual approach to integrated reporting is based on integrated thinking. A systematic approach in the disclosure of non-financial information creates additional opportunities to analyze and improve the various aspects of the economic, environmental and social development for the Russian companies. For this purpose, the development of the detailed legislation background in integrated reporting is required.
\end{abstract}

Keywords: non-financial reporting, sustainable development, integrated reporting, intellectual capital, Global Reporting Initiative (GRI)

JEL: G30, Q56, Z00 


\section{References}

1. Arrington C.E., Francis J.R. Letting the chat out of the bag: Deconstruction, privilege and accounting research. Accounting, Organizations and Society. 1989;14(1-2):128. DOI: 10.1016/0361-3682(89)90030-5

2. Arrington C.E., Francis J.R. Giving economic accounts: Accounting as cultural practice. Accounting, Organizations and Society. 1993;18(2-3):107-124. DOI: 10.1016/0361-3682(93)90029-6

3. Arrington C.E., Schweiker W. The rhetoric and rationality of accounting research. Accounting, Organizations and Society. 1992;17(6):511-533. DOI: 10.1016/0361-3682(92)90011-G

4. Baker M., Schaltegger S. Pragmatism and new directions in social and environmental accountability research. Accounting, Auditing \& Accountability Journal. 2015;28(2):263-294. DOI: 10.1108/AAAJ-08-2012-01079

5. Borzakov D.V. Institutionalization of corporate non-financial reporting in Russia and abroad. Vestnik Voronezhskogo gosudarstvennogo universiteta. Seriya: Ekonomika i upravlenie $=$ Proceedings of Voronezh State University. Series: Economy and Management. 2015;(3):58-66. (In Russ.).

6. Brown J., Dillard J. Opening accounting to critical scrutiny: Towards dialogic accounting for policy analysis and democracy. Journal of Comparative Policy Analysis: Research and Practice. 2015;17(3):247-268. DOI: $10.1080 / 13876988.2014 .989684$

7. Buhr N., Gray R., Milne M.J. Histories, rationales, voluntary standards and future prospects for sustainability reporting: CSR, GRI, IIRC and beyond. In: Bebbington J., Unerman J., O’Dwyer B., eds. Sustainability accounting and accountability. Abingdon, New York: Routledge; 2014:51-71.

8. Burritt R.L., Schaltegger S. Sustainability accounting and reporting: Fad or trend? Accounting, Auditing \& Accountability Journal. 2010;23(7):829-846. DOI: $10.1108 / 09513571011080144$

9. Chambers R.J. The poverty of accounting discourse. Abacus. 1999;35(3):241-252. DOI: 10.1111/14676281.00044

10. Contrafatto M., Thomson I., Monk E.A. Peru, mountains and los niños: Dialogic action, accounting and sustainable transformation. Critical Perspectives on Accounting. 2015;33:117-136. DOI: 10.1016/j. cpa.2015.04.009

11. Corporate Register. 2018. URL: http://www. corporateregister.com

12. Corporate social responsibility and reporting: Present and future. Analytical review of corporate nonfinancial reports 2015-2016. Moscow: Russian Union of Industrialists and Entrepreneurs (RSPP); 2017. 140 p.
URL: http://media.rspp.ru/document/1/7/4/743222fc4c 6650093518c635d0e8ecdd.pdf (accessed 7.03.2018). (In Russ.).

13. Deegan C. Twenty five years of social and environmental accounting research within Critical Perspectives of Accounting: Hits, misses and ways forward. Critical Perspectives on Accounting. 2017;43:6587. DOI: $10.1016 /$ j.cpa.2016.06.005

14. Directive 2014/95/EU of the European Parliament and of the Council of 22 October 2014. URL: http://eur-lex.europa.eu/legal-content/EN/ TXT/?uri=CELEX\%3A32014L0095

15. Feruleva N.V., Ivashkovskaya I.V. Assessment of intellectual capital influence on corporate value as a field for further investigations in corporate finance. Korporativnye finansy $=$ Journal of Corporate Finance Research. 2018;15(1):64-76.

16. Forbes. 2016. URL: http://www.forbes.ru

17. Global Reporting Initiative (GRI). 2018. URL: https:// www.globalreporting.org

18. Gray R., Brennan A., Malpas J. New accounts: Towards a reframing of social accounting. Accounting Forum. 2014;38(4):258-273. DOI: 10.1016/j.accfor.2013.10.005

19. Gray R. Back to basics: What do we mean by environmental (and social) accounting and what is it for? - A reaction to Thornton. Critical Perspectives on Accounting. 2013;24(6):459-468. DOI: 10.1016/j. cpa.2013.04.005

20. Gray R. Reading for displeasure: Why bother with social accounting at all? Social and Environmental Accountability Journal. 2016;36(2):153-161. DOI: 10.1080/0969160X.2016.1197625

21. G20 green finance synthesis report 2017. URL: http:// unepinquiry.org/wp-content/uploads/2017/07/2017_ GFSG_Synthesis_Report_EN.pdf

22. Harvey B. Social development will not deliver social licence to operate for the extractive sector. The Extractive Industries and Society. 2014;1(1):7-11. DOI: 10.1016/j. exis.2013.11.001

23. Higgins C., Coffey B. Improving how sustainability reports drive change: A critical discourse analysis. Journal of Cleaner Production. 2016;136(Pt. A):18-29. DOI: 10.1016/j.jclepro.2016.01.101.

24. International Integrated Reporting Council (IIRC). 2018. URL: http://integratedreporting.org

25. International Organization for Standardization (ISO). 2018. URL: https://www.iso.org/

26. Ivashkovskaya I.V., Stepanova A.N., Eliseeva N. Does corporate financial architecture contribute to sustainable corporate growth? The evidence from Russian companies. Korporativnye finansy $=$ Journal of Corporate Finance Research. 2014;8(4):11-33. 
27. Kuzubov S.A. Sustainability: A new paradigm of corporate reporting. Korporativnye finansy $=$ Journal of Corporate Finance Research. 2017;14(4):7-10.

28. Lehman G., Kuruppu S.C. A framework for social and environmental accounting research. Accounting Forum. 2017;41(3):139-146. DOI: 10.1016/j.accfor.2017.07.001

29. Lehman G. Moral will, accounting and the phronemos. Critical Perspectives on Accounting. 2014;25(3):210-216. DOI: 10.1016/j.cpa.2013.10.004

30. Lehman G. Charles Taylor's ecological conversations: politics, commonalities and the natural environment. London: Palgrave Macmillan; 2015. 201 p.

31. Lehman G. The language of environmental and social accounting research: The expression of beauty and truth. Critical Perspectives on Accounting. 2017;44:30-41. DOI: 10.1016/j.cpa.2016.11.005

32. Lev B., Gu F. The end of accounting and the path forward for investors and managers. Hoboken, NJ: John Wiley \& Sons, Inc.; 2016. 288 p.

33. Makarov I. Climate of the future: why Russia should ratify the Paris Agreement. 2016. URL: http://www.rbc. ru/opinions/economics/04/11/2016/581b6a5c9a794757 $4 f 524263$ (In Russ.).

34. Milne M.J., Gray R.H. W(h)ither ecology? The triple bottom line, the global reporting initiative, and corporate sustainability reporting. Journal of Business Ethics. 2013;118(1):13-29. DOI: 10.1007/s10551-012-1543-8

35. Non-financial reporting of companies: In pursuit of success. Infromation bulletin. Clean technologies and sustainable development. 2017;(2). URL: http://www. ey.com/Publication/vwLUAssets/EY-ccass-newslettermay-2017/\$File/EY-ccass-newsletter-may-2017.pdf (accessed 16.02.2018).(In Russ.).

36. Owen D. Chronicles of wasted time?: A personal reflection on the current state of, and future prospects for, social and environmental accounting research. Accounting, Auditing \& Accountability Journal. 2008;21(2):240-267. DOI: 10.1108/09513570810854428

37. Parker L.D. Building bridges to the future: Mapping the territory for developing social and environmental accountability. Social and Environmental Accountability Journal. 2011;31(1):7-24. DOI: 10.1080/0969160X.2011.556389

38. RIA Rating. 2017. URL: http://www.riarating.ru/ infografika/20160127/630007042.html (In Russ.).

39. Russian Regional Network (RRS) for integrated reporting. 2018. URL: http://ir.org.ru/regionalnaya-set (In Russ.).

40. Russian Union of Industrialists and Entrepreneurs (RSPP). 2018. URL: http://eng.rspp.ru/
41. Spence C. Social accounting's emancipatory potential: A Gramscian critique. Critical Perspectives on Accounting. 2009;20(2):205-227. DOI: 10.1016/j. cpa.2007.06.003

42. Spence C., Chabrak N., Pucci R. Doxic sunglasses: A response to "Green accounting and Green Eyeshades: Twenty years later". Critical Perspectives on Accounting. 2013;24(6):469-473. DOI: 10.1016/j.cpa.2013.05.002

43. Sustainability Accounting Standards Board (SASB). 2018. URL: https://www.sasb.org/

44. Sustainable Stock Exchanges Initiative (SSE Initiative). 2018. URL: http://www.sseinitiative.org

45. The Concept of the development of public nonfinancial reporting and the plan of measures to implement the Concept of the development of public non-financial reporting. Approved by the decree of the Government of the Russian Federation of 05.05.2017 № 876-p. URL: http://docs.cntd.ru/document/456064017 (accessed 17.03.2018). (In Russ.).

46. The road ahead. The KPMG survey of corporate responsibility reporting 2017. URL: https://assets.kpmg. com/content/dam/kpmg/xx/pdf/2017/10/kpmg-surveyof-corporate-responsibility-reporting-2017.pdf

47. Thomson I. 'But does sustainability need capitalism or an integrated report' a commentary on 'The International Integrated Reporting Council: A story of failure' by Flower, J. Critical Perspectives on Accounting. 2015;27:1822. DOI: $10.1016 /$ j.cpa.2014.07.003

48. Tinker T., Neimark M., Lehman C. Falling down the hole in the middle of the road: Political quietism in corporate social reporting. Accounting, Auditing \& Accountability Journal. 1991;4(2):28-54. DOI: $10.1108 / 09513579110000504$

49. United Nations Development Programme (UNDP). 2018. URL: http://www.undp.org/content/undp/en/home/ sustainable-development-goals.html

50. Ursul A.D. Conceptual problems of sustainable development. Ispol'zovanie i okhrana prirodnykh resursov v Rossii. 2005;(1):30-38. (In Russ.).

51. Walker S.P. Revisiting the roles of accounting in society. Accounting, Organizations and Society. 2016;49:41-50. DOI: 10.1016/j.aos.2015.11.007

52. 2013 Six growing trends in corporate sustainability. An EY survey in cooperation with GreenBiz Group. URL: https://www.ey.com/Publication/ vwLUAssets/Six_growing_trends_in_corporate_ sustainability_2013/\%24FILE/Six_growing_trends_in_ corporate_sustainability_2013.pdf 